\title{
FATORES DE RISCO PARA OCORRÊNCIA DE ERRO NO PREPARO DE MEDICAMENTOS ENDOVENOSOS: UMA REVISÃO INTEGRATIVA
}

Flavia Giron Camerini ${ }^{1}$, Andressa de Paiva Colcher ${ }^{2}$, Daniela dos Santos Moraes ${ }^{3}$, Danielle Lemos de Souza ${ }^{4}$, Juliana Rodrigues de Vasconcelos ${ }^{5}$, Rodrigo de Oliveira Neves ${ }^{6}$

Enfermeira. Mestre em Enfermagem. Hospital Pro-Cardíaco do RJ. Niterói-RJ-Brasil.

2Enfermeira. Hospital Miguel Couto. Rio de Janeiro-RJ-Brasil.

${ }^{3}$ Enfermeira. Hospital Infantil Ismélia da Silveira. Rio de Janeiro-RJ-Brasil.

${ }^{4}$ Enfermeira. Hospital Pró-cardíaco do Rio de Janeiro. Itaboraí-RJ-Brasil.

${ }^{5}$ Enfermeira. Hospital Federal de Bonsucesso. Rio de Janeiro-RJ-Brasil.

${ }^{6}$ Enfermeiro. Rio de Janeiro-RJ-Brasil.

RESUMO: Estudo cujo objetivo foi identificar e analisar os fatores de risco para a ocorrência de erros no preparo de medicamentos endovenosos pela enfermagem e propor um checklist para promover maior segurança no preparo. Foram selecionados treze artigos das bases de dados LILACS, BDENF, SciELO e MEDLINE/PUBMED entre os períodos de 2004 a 2013. Destacaram-se quatro principais categorias: fatores psicológicos e sobrecarga de trabalho, erros relacionados ao ambiente de preparo, déficit de atualização em educação em saúde e erros relacionados à técnica de preparo. Conclui-se que a técnica de preparo foi o fator de risco mais citado nos estudos, seguido das categorias déficit de atualização em educação e saúde, ambientes de preparo e sobrecarga de trabalho associada a fatores psicológicos. Baseado nas dificuldades identificadas nas categorias encontradas foi elaborado um checklist relacionado à técnica de preparo, visando minimizar erros e garantir a qualidade da assistência e segurança do paciente.

DESCRITORES: Enfermagem; Erros de medicação; Fatores de risco.

\section{RISK FACTORS FOR THE OCCURRENCE OF ERRORS INTHE PREPARATION OF INTRAVENOUS MEDICATIONS: AN INTEGRATIVE REVIEW}

\begin{abstract}
This study aimed to identify and analyze the risk factors for the occurrence of errors in the preparation of intravenous medications by nurses, and to propose a checklist in order to promote greater safety in the preparation. A total of 13 articles were selected from the LILACS, BDENF, SciELO and MEDLINE/PUBMED databases, published in the period 2004 - 2013. Four main categories stood out: psychological factors and work overload, errors related to the preparation environment, lack of updating in health education, and errors related to the preparation technique. It is concluded that the preparation technique was the most-mentioned risk factor in the studies, followed by the categories of lack of updating in health and education, preparation environment, and work overload associated with psychological factors. Based in the difficulties identified in the categories found, a checklist was developed related to the preparation technique, with a view to minimizing errors and ensuring quality of care and patient safety.
\end{abstract}

DESCRIPTORS: Nursing; Medication errors; Risk factors.

\section{FACTORES DE RIESGO PARA OCURRENCIA DE ERRORESENLAPREPARACIÓNDEMEDICAMENTOS ENDOVENOSOS: UNA REVISIÓN INTEGRATIVA}

RESUMEN: Estudio cuyo objetivo fue identificar y analizar los factores de riesgo para la ocurrencia de errores en la preparación de medicamentos endovenosos por la enfermería y proponer un checklist para promover más seguridad en esa preparación. Fueron seleccionados trece artículos de las bases de datos LILACS, BDENF, SciELO y MEDLINE/PUBMED entre los periodos de 2004 a 2013. Se destacaron cuatro principales categorías: factores psicológicos y sobrecarga de trabajo, errores asociados al ambiente de preparación, déficit de actualización en educación en salud y errores referentes a la técnica de preparación. Se concluye que la técnica de preparación fue el factor de riesgo más mencionado en los estudios, seguido de las categorías déficit de actualización en educación y salud, ambientes de preparación y sobrecarga de trabajo asociada a factores psicológicos. Con base en las dificultades identificadas en las categorías halladas, fue elaborado un checklist para la técnica de preparación, con la finalidad de minimizar errores y garantizar la cualidad de la asistencia y seguridad del paciente.

DESCRIPTORES: Enfermería; Errores de medicación; Factores de riesgo.
Autor Correspondente:

Flávia Giron Camerini

Hospital Pró-Cardíaco

Rua Marechal Raul de Albuquerque, 122 - 24370-025 - Niterói-RJ-Brasil

E-mail: fcamerini@gmail.com
Recebido: 06/19/2013

Finalizado: 03/03/2014 


\section{INTRODUÇÃO}

Atualmente estima-se que cerca de $30 \%$ dos danos durante a hospitalização estão associados a erros com medicamentos, os quais trazem sérias consequências econômicas às instituições de saúde ${ }^{(1)}$. A American Society of Health-System Pharmacists (ASHP) classifica erro de medicação, segundo a sua natureza, em doze tipos, dentre eles está o erro de preparo de medicamentos ${ }^{(2)}$.

A Agência Nacional de Vigilância Sanitária (ANVISA), em sua Resolução de Diretoria Colegiada (RDC) n. $45^{(3)}$, define preparo como o ato de misturar medicamentos destinados ao uso endovenoso, empregando-se técnicas que assegurem sua integridade microbiológica e seu equilíbrio físico-químico. Essa mesma resolução aponta o enfermeiro com sendo um dos profissionais responsáveis pelo preparo das soluções administradas por via endovenosa.

Os medicamentos endovenosos são muito utilizados e apresentam maior potencial de dano ao paciente, por esse fato, erros com medicamentos por essa via devem ser prevenidos ${ }^{(4)}$. Nesse sentido, é imperativo que o enfermeiro identifique os fatores de risco relacionados à segurança e à prevenção de ocorrências de erros no preparo de medicamentos endovenosos.

Neste contexto, apesar de já existirem publicações a respeito dessa temática, é possível observar que faltam publicações acerca da atuação do enfermeiro, especificamente na identificação dos fatores de risco no preparo de medicamentos.

Em um estudo internacional realizado em três diferentes países observou-se que, das 824 doses preparadas, a rotulagem estava ausente ou incorreta de 20 a 99\%, o diluente foi utilizado erradamente de 1 a 49\%, e houve ao menos um desvio da técnica asséptica em 19 a 100\% ${ }^{(5)}$.

Estudos brasileiros também apontam a incidência de erros com medicamentos. Em um estudo, de 8.152 fármacos analisados, foram identificados 1.717 erros de doses ou diluições. Outro estudo nacional, realizado em unidades de tratamento intensivo mostrou que medicamentos foram responsáveis por $78 \%$ dos erros graves, mais comumente causados por erro de dose. Entre eles, $11 \%$ acarretariam risco de morte ${ }^{(5)}$.

Durante o estudo da temática, surgiu o seguinte questionamento: Quais os principais fatores de risco para a ocorrência de erros no preparo de medicamentos endovenosos pela equipe de enfermagem no ambiente hospitalar?

Portanto, o objeto de estudo deste trabalho é o preparo de medicamentos endovenosos para pacientes adultos visando à análise e a prevenção dos riscos envolvidos.

Buscando contribuir para a otimização do cuidado de enfermagem e segurança do paciente, este estudo tem como objetivo identificar e analisar na literatura os fatores de risco para a ocorrência de erros no preparo de medicamentos pela enfermagem e propor um checklist a fim de promover maior segurança no preparo.

\section{METODOLOGIA}

A revisão integrativa foi o método utilizado para a elaboração do presente trabalho, no qual foram selecionados artigos, por meio de pesquisa virtual, nas bases de dados LILACS (Literatura LatinoAmericana e do Caribe em Ciências de Saúde), BDENF (Base de Dados Bibliográficos Especializada na Área de Enfermagem), SciELO (Scientific Electronic Library Online) e MEDLINE/PUBMED (Medical Literature Analysis and Retrieval System on-line).

Foi empregado o vocabulário estruturado e trilíngue DeCS/BVS: enfermagem, erros de medicação, via de administração de medicamentos, infusões intravenosas, segurança do paciente, estabilidade de medicamentos e fatores de risco. Destacase que foi utilizado, entre os descritores, o operador booleano AND. Para a seleção dos estudos foram aplicados os seguintes critérios de inclusão: publicação nas bases de dados selecionadas, no idioma português, em formato de artigo, disponível gratuitamente e em texto completo, dos anos compreendidos entre 2004 e 2013, com abordagem no preparo de medicamentos endovenosos, em adultos em unidade hospitalar.

As publicações encontradas foram previamente selecionadas pelo título e resumo e, as que contemplaram os critérios de inclusão para realização, foram avaliadas na íntegra. Em se tratando de um estudo de revisão com base virtual, foram obedecidos os preceitos éticos citando os autores dos artigos e periódicos analisados. 


\section{RESULTADOS}

A partir dos descritores previamente definidos obteve-se 660 estudos, conforme indicado na Tabela 1 , onde se podem identificar os resultados encontrados a partir da busca, através do cruzamento do descritor "Enfermagem" com os demais, conforme a biblioteca virtual e bases de dados selecionadas para pesquisa.

Após a análise dos dados da Tabela 1, dos 660 artigos, 575 referências apresentavam-se em idioma nacional. Os estudos em duplicidade foram suprimidos à organização dos dados da publicação. Após a sobreposição das referências encontradas, foram excluídas 26 publicações repetidas, totalizando 549 artigos. Foram excluídos os artigos que não estavam disponíveis na íntegra de forma gratuita e que não tinham como tema central o preparo de medicamentos endovenosos, em adultos em unidade hospitalar. Após a leitura e análise do título e resumo dessa amostra final, 13 publicações apresentaram conceitos relevantes para o alcance do objetivo desta pesquisa e foram analisadas.

Tabela 1 - Distribuição dos estudos encontrados, em frequência absoluta, por bases de dados. Rio deJaneiro-RJ-Brasil, 2012

\begin{tabular}{lcccc}
\hline Descritores & LILACS & SCIELO & BDENF & $\begin{array}{l}\text { Total por } \\
\text { descritor }\end{array}$ \\
\hline $\begin{array}{l}\text { Enfermagem } \\
\text { medicamentos }\end{array}$ & 08 & 02 & 06 & 16 \\
\hline $\begin{array}{l}\text { Enfermagem AND Vias de Administração de } \\
\text { medicamentos }\end{array}$ & 09 & 03 & 06 & 18 \\
\hline Enfermagem AND Erros de medicação & 104 & 27 & 78 & 209 \\
\hline Enfermagem AND Segurança do paciente & 190 & 49 & 137 & 376 \\
\hline Enfermagem AND Infusão intravenosa & 22 & 03 & 16 & 41 \\
\hline Total por base de dados & 333 & 84 & 243 & 660 \\
\hline
\end{tabular}

\section{DISCUSSÃO}

Dos 13 estudos que preencheram os critérios de inclusão desta revisão bibliográfica, emergiram quatro principais fatores de risco relacionados ao preparo de medicamentos endovenosos que foram utilizados como categorias de análise: fatores psicológicos e sobrecarga de trabalho ${ }^{(6-8)}$, erro relacionado ao ambiente de preparo $^{(9-11)}$, déficit de atualização em educação e saúde ${ }^{(12-15)}$ e erro relacionado á técnica de preparo e a manipulação do medicamento ${ }^{(6-7,9,13,16-18)}$.

\section{Fatores Psicológicos e Sobrecarga de Trabalho}

Dos 10 artigos analisados, três apontaram aspectos relevantes relacionados a essa categoria.

Em um estudo ${ }^{(6)}$, com abordagem metodológica quanti-descritiva que teve como objetivo identificar os fatores que contribuíam para a ocorrência de erro relacionado ao preparo de medicamentos. Os fatores identificados foram "falta de atenção", "relação profissional/paciente" e "excesso de trabalho", que totalizaram mais de
70\% das causas de erros identificados.

Embasando o artigo analisado, no que diz respeito ao aspecto "falta de atenção", um estudo $^{(1)}$, realizado em quatro hospitais, também aponta esse mesmo fator como sendo o mais citado pelos entrevistados.

Outro estudo analisado nesta categoria ${ }^{(7)}$, conduzido em duas instituições hospitalares em São Paulo, verificou-se que 37\% dos erros ocorreram no período da tarde e $35 \%$ pela manhã, sendo percentagem menor à noite (de $29 \%$ ). As diferenças observadas entre os turnos são justificadas pelo excesso de trabalho diurno associado à necessidade do cumprimento das rotinas, maior número de funcionários, manipulação dos prontuários, barulho gerando cansaço e falta de atenção dos profissionais.

Dos artigos selecionados, um deles ${ }^{(8)}$ aborda a sobrecarga de trabalho dos técnicos de enfermagem, como a principal problemática no preparo de medicamentos. Os resultados indicam que os erros mais comuns estão relacionados à preparação dos medicamentos, e as justificativas para a ocorrência de erros evidenciam a sobrecarga de trabalho e a falta de atenção. 
Assim como relatado nos estudos ${ }^{(7-8)}$, o estudo $^{(19)}$ aborda a sobrecarga de trabalho como um fator comumente citado nos artigos, neste estudo $^{(19)}$, foram realizadas entrevistas com técnicos de enfermagem no qual a sobrecarga de trabalho foi um dos fatores de risco mais prevalentes.

Com isso, é possível observar que o excesso de trabalho é o fator de risco mais citado nos artigos como um fator desencadeante de estresse, falta de atenção e cansaço que promovem um ambiente facilitador para a ocorrência de erros.

\section{Erros relacionados ao ambiente de preparo}

Dos artigos selecionados, três relacionam o ambiente como fator de risco para a ocorrência de erros.

Em um estudo ${ }^{(9)}$ relacionado aos fatores que interferem no preparo dos medicamentos, destacase que o barulho, a desorganização, iluminação, ventilação, circulação de pessoas, interferem no preparo de medicamentos. No mesmo artigo a autora cita que os erros relacionados ao ambiente $(45,9 \%)$ corresponderam ao maior percentual dos erros analisados.

No artigo ${ }^{(10)}$, os ruídos no local de preparo, a grande circulação de pessoas no posto de enfermagem e a conversa paralela durante o preparo foram fatores que evidenciaram a probabilidade de ocorrência de erros.

Outro estudo ${ }^{(11)}$ que se refere a erros relacionados ao ambiente que cita os fatores ambienteis que predispõem à distração da equipe de enfermagem durante o preparo e a administração de medicamentos. No qual cita que foram identificados 100 fatores que poderiam causar distrações da enfermagem predispondo seu erro, entre eles, a presença de telefone fixo da instituição ou celular tocando, interrupção por outros profissionais, mudanças não padronizadas de prescrições médicas, sobreposição de diferentes tarefas pelo mesmo profissional nos horários padronizados para medicação e ausência de planejamento formal da assistência ${ }^{(11)}$.

Um estudo ${ }^{(20)}$ que corrobora com as pesquisa supracitadas, aponta o ambiente de preparo ruidoso compreendendo $68 \%$ das situações facilitadoras para o erro com medicamentos endovenosos. Ambiente com mais de uma pessoa no local do preparo correspondeu 25\% dos fatores de risco para erro e interrupções constantes também foram evidenciadas nesse estudo em $33 \%$. As interrupções para esclarecimento de dúvidas ou atendimento ao telefone foram referidas em $15 \%$ dos casos.

A Resolução da Diretoria Colegiada - RDC 45, expedida pela ANVISA ${ }^{(3)}$ determina que a iluminação e ventilação devam ser suficientes para que a temperatura e a umidade do ar não deteriorem os medicamentos e facilitem as atividades desenvolvidas. $\mathrm{O}$ acesso ao ambiente de preparo das soluções endovenosas deve ser restrito aos profissionais diretamente envolvidos, garantindo assim uma maior segurança e minimizando os fatores de risco.

Em artigo, que postula que o erro é característica imutável deste ser, sendo necessária a transformação do ambiente no qual os seres humanos agem a fim de promover um ambiente onde seja difícil errar ${ }^{(21)}$.

\section{Déficit de atualização em educação em saúde}

Nessa categoria, quatro artigos enfatizaram a educação permanente como uma ferramenta indispensável para a promoção de uma prática segura.

Os profissionais de enfermagem identificaram algumas dificuldades nas atividades diárias, dentre elas o preparo de medicamentos endovenosos ${ }^{(12)}$. Pesquisa também trata da necessidade do conhecimento dos profissionais, abordando de forma mais específica, antibioticoterapia, já que falhas durante o preparo de antibióticos podem levar ao insucesso terapêutico e ao aumento da resistência bacteriana ${ }^{(13)}$.

Em um estudo ${ }^{(14)}$ que aponta as principais dúvidas dos profissionais referiam-se ao tipo de frasco, tempo de troca da solução e concentração adequada. Um fator que contribuiu para o insucesso da terapêutica foi a utilização de equipos não específicos para a administração de insulinoterapia, favorecendo o aumento da adsorção do medicamento. Com isso, verifica-se a necessidade do conhecimento do profissional em relação ao material a ser utilizado durante o preparo de medicamentos endovenosos.

Em um estudo realizado em um hospital universitário ${ }^{(15)}$ por um período de seis anos, foram 
descritas as atividades passivas realizadas por um Centro de Informações sobre Medicamentos. Das 8.035 solicitações recebidas por este centro, 45\% das dúvidas foram advindas dos profissionais de enfermagem, visto que esses são responsáveis por várias etapas do processo de medicação. Isso demonstra que a necessidade do fornecimento de informações rápidas e atualizadas a esses profissionais contribui de forma significativa para prevenção de erros e promoção do uso seguro e racional de medicamentos.

A importância de programas educacionais destacados no estudo(22) alerta para que seja voltado para cada realidade institucional, pois uma rotina pré-estabelecida pode não conseguir alcançar resultados satisfatórios. Portanto, a técnica de preparo de medicamentos endovenosos deve ser acompanhada de forma continua a fim de promover uma prática segura.

\section{Erros relacionados à técnica de preparo}

Para que os medicamentos endovenosos sejam utilizados com segurança é indispensável atender a requisitos mínimos durante o seu preparo com intuito de manter suas características físico-químicas, estabilidade, esterilidade bem como interações e incompatibilidades medicamentosas $^{(3)}$. Neste contexto, para esta categoria, foram selecionados sete trabalhos que identificaram erros relacionados à técnica de preparo de medicamentos endovenosos.

O preparo antecipado da solução foi identificado como erro em dois destes estudos ${ }^{(9,16)}$, sendo que em um desses a ocorrência foi superior a 50\%. A autora relaciona essa ocorrência à grande quantidade de medicamentos aprazados para um mesmo horário(9).

Devido a grande variabilidade de medicamentos o tempo de estabilidade após a reconstituição é diferenciado, sugere-se que o preparo seja efetuado imediatamente antes da administração a fim de minimizar a possibilidade de erros e possível diminuição do efeito terapêutico.

Em um dos estudos desta categoria, diluição e doses inadequadas somaram quase 30\% dos erros $^{(6)}$. Já em uma pesquisa ${ }^{(13)}$ acerca de antibioticoterapia é ratificada a importância da quantidade de diluente necessário para a efetividade terapêutica de cada medicamento de acordo com as suas características farmacológicas.

Em um estudo ${ }^{(7)}$ realizado em duas instituições hospitalares em São Paulo objetivou-se caracterizar erros e avaliar consequências na gravidade dos pacientes e carga de trabalho de enfermagem antes e após o erro. Do total de 52 erros, $21,15 \%$ estavam relacionados ao preparo do medicamento errado.

No que diz respeito à técnica de higienização das mãos antes do preparo dos medicamentos, o artigo $^{(9)}$ verificou ausência deste procedimento em dois dos quatro hospitais investigados e o artigo ${ }^{(15)}$ obteve 29,2\% de erro na avaliação deste mesmo índice.

Ausência de desinfecção das ampolas e/ ou frasco-ampolas foi identificada em três estudos $^{(9,13,16)}$ variando entre 57 a $80 \%$. As diretrizes do Centers for Disease Control and Prevention informam que neste caso, o risco de contaminação é mínimo, mas quando ocorrem podem oferecer risco de morte ao paciente ${ }^{(23)}$.

Portanto, é necessário seguir as recomendações internacionais ${ }^{(23)}$ que preconizam que a desinfecção deste tipo de material deve ser realizada por meio da fricção com álcool a 70\% por três vezes antes do uso.

A observação da bancada onde eram preparados os medicamentos foi realizada nos estudos $^{(16,20)}$. Evidenciaram que não era realizada a desinfecção da bancada com álcool a 70\% em aproximadamente $77 \%$ do número de doses observadas $^{(16)}$, já no estudo ${ }^{(20)}$ constatou-se que a bancada não estava seca em 33\%.

É importante que a rotina de desinfecção de superfícies seja orientada pela Comissão de Controle de Infecção Hospitalar a fim de que haja um controle do número de microrganismos presentes na bancada, que pode chegar, com esse tipo de precaução, a ser reduzido em cerca de $90 \%{ }^{(16,24-25)}$.

O ultimo artigo analisado nesta categoria aponta que e falta de conhecimento técnico foi uma das fragilidades que facilitam a ocorrência de erro durante o preparo e a administração de medicamento $^{(18)}$.

O preparo antecipado, a reconstituição e diluição inadequadas, ausência de desinfecção das ampolas e bancadas e a omissão da etapa de higienização das mãos foram os principais fatores de risco identificados nessa categoria.

Cabe ressaltar que a comparação desses 
estudos foi dificultada devido à valorização de diferentes informações pelos autores e o tipo de abordagem metodológica utilizada. Portanto, nota-se a necessidade da criação e validação de um checklist específico para a etapa de preparo de medicamentos endovenosos para melhores comparações de estudos observacionais. Nesse sentido, foi elaborado um checklist procurando orientar os aspectos importantes a serem observados na etapa do preparo, que se encontra ao final do texto.

\section{CONCLUSÃO}

Os medicamentos endovenosos são amplamente utilizados na prática profissional e merecem especial atenção no que se refere ao seu preparo.

Os principais fatores de risco para a ocorrência de erros evidenciados neste estudo foram a sobrecarga de trabalho associada a fatores psicológicos, déficit de atualização em educação e saúde e manipulação incorreta dos medicamentos em ambiente inadequado.

Sobrecarga de trabalho associada a fatores psicológicos e ambiente de preparo apresentaramse em um total de quatro artigos. A categoria de déficit de atualização em educação em saúde alcançou este mesmo quantitativo durante a análise dos estudos, o que ressaltou a relevância desta dinâmica nas instituições de saúde.

As pesquisas que abordaram a técnica de preparo apresentaram-se em seis artigos, com abordagens distintas referentes aos critérios de avaliação desta etapa do processo de medicação.

A utilização de um instrumento que possibilite a comparação dos resultados poderá promover um alicerce para que a educação em saúde direcione treinamentos baseados nas melhores evidências científicas disponíveis com consequente crescimento da qualidade da assistência de enfermagem.

\section{REFERÊNCIAS}

1. Miasso A, Grou C, Cassiani S, Silva A, Fakih F. Erros de medicação: tipos, fatores causais e providências tomadas em quatro hospitais brasileiros. Rev Esc Enferm USP. 2006;40(4):524-32.
2. American Society of Hospital Pharmacists. ASHP guidelines on preventing medication errors in hospitals. Am J Hosp Pharm. 1993;(50):305-14.

3. Agência Nacional de Vigilância Sanitária (BR). Resolução RDC n. 45 de 12 de março de 2003. Dispõe sobre o Regulamento Técnico de Boas Práticas de Utilização das Soluções Parenterais (SP) em Serviços de Saúde.Brasília (DF): MS; 2003.

4. Cortez EA, Soares GRS, Silva ICM, Carmo TGD. Preparo e administração venosa de medicamentos e soros sob a ótica da Resolução COFEN n. 311/07. Acta Paul. Enferm. 2010;23(6):843-51.

5. Organização Pan-Americana da Saúde/Organização Mundial da Saúde. Erros: evitando o evitável. Uso Racional de Medicamentos: Temas Selecionados. Representação do Brasil e do Departamento de Assistência Farmacêutica e Insumos Estratégicos da Secretaria de Ciência,Tecnologia e Insumos Estratégicos do Ministério da Saúde; 2005.

6. Praxedes MFS, Filho PCPT. Erros e Ações Praticadas pela Instituição Hospitar no Preparo e Administração de Medicamentos. Reme. 2011;15(3): 406-11.

7. Toffoleto MC, Padilha KG. Consequências de Medicação em Unidades de Terapia Intensiva e Semiintensiva. Rev Esc Enferm USP. 2006;40(2):247-52.

8. Lopes BC, Vargas MAO, Azeredo NSG, Behenck A. Erros de medicação realizados pelo técnico de enfermagem na UTI: contextualização da problemática. Enfermagem em Foco 2012;3(1):16-21

9. Miasso Al, Silva AEBC, Cassiani SHB, Grou CR, Oliveira RC, Fakih FT. O processo de preparo e administração de medicamentos: identificação de problemas para propor melhorias e prevenir erros de medicação. Rev. Latino-Am. Enfermagem. 2006;14(3):354-63.

10. Oliveira RB, Melo ECP. O sistema de medicação em um hospital especializado no município do Rio de Janeiro. Esc. Anna Nery. 2011;15(3):480-9.

11. Lemos NRF, Silva VR, Martinez MR. Fatores que predispõem à distração da equipe de enfermagem durante o preparo e a administração de medicamentos. Reme. 2012;16(2):201-7.

12. Silva LD, Camerini FG. Análise da administração de medicamentos intravenosos em hospital da rede sentinela. Texto Contexto Enferm.2012;21(3):633-41.

13. Hoefel HHK, Lautert L. Administração endovenosa de antibióticos e resistência bacteriana: responsabilidade da enfermagem. Rev. Eletr. Enf. 2006; 8(3):441-9.

14. Lima SA, Andreoli RLF, Grossi SAA, Secoli SR. Insulina intravenosa: controvérsias sobre o processo de adsorção nos dispositivos de infusão. Rev. Gaúcha Enferm. 2008;29(2):292-300. 
15. Santos L, Jacoby T, Zuckermann J, Martinbiancho JK, Mahmud SDP, Negretto GW, et al. Centro de informações sobre medicamentos: avaliação das informações passivas em hospital universitário no sul do Brasil. Rev. HCPA. 2009;29(3):212-7.

16. Camerini FG, Silva LD. Segurança do paciente: análise do preparo de medicação intravenosa em hospital da rede sentinela. Texto Contexto Enferm. 2011;20(1):41-9.

17. Rodrigues MCS, Oliveira LC. Erros na administração de antibióticos em unidade de terapia intensiva de hospital de ensino. Rev. Eletr. Enf. 2010;12(3):511-9

18. Lolita Dopico da Silva LD, Passos RS, Carvalho MF. Características e evidências da produção científica de enfermeiros sobre erros de medicação no ambiente hospitalar. Rev Rene. 2012;13(2):480-91

19. Corbellini VL, Schilling MCL, Frantz SF, Godinho TG, Urbanetto JS. Eventos adversos relacionados a medicamentos: percepção de técnicos e auxiliares de enfermagem. Rev. bras. Enferm. 2011;64(2):241-7.

20. Melo ABR, Silva LD. Segurança na terapia medicamentosa:uma revisão bibliográfica. Esc. Anna Nery. 2008;12(1):166-72.

21. Pedreira MLG. Práticas de enfermagem baseadas em evidências para promover a segurança do paciente. Acta Paul. Enferm. 2009;22:880-1.

22. Praxedes MFS, Filho PCPT. Identificação de erros no preparo e administração demedicamentos pela equipe de enfermagem e das ações praticadaspela instituição hospitalar. Cogitare enferm.2008;13(4):514-9.

23. Silva MF, Conceição FA, Leite MMJ. Educação continuada: um levantamento de necessidades da equipe de enfermagem. Mundo Saúde. 2008;32(1):4755.

24. O'Grady NP, Alexander M, Burns LA, Dellinger EP, Garland J. Guidelines for the prevention of intravascular catheter-related infection. Clin Infect Dis.2011;52:162-93.

25. Instituto Nacional de Câncer (INCA). Ações de enfermagem para o controle do câncer: uma proposta de integração ensino-serviço. $3^{a}$ ed. Rio de Janeiro; 2008.

\section{LISTA DE VERIFICAÇÃO PARA A PREPARAÇÃO DE} MEDICAMENTOS INTRAVENOSOS

1. Prepare o ambiente, de modo a evitar interrupções durante a preparação do medicamento e reduzir o excesso de ruído na área.

2. Evite mais de um profissional na sala de preparação

3. Verificar e controlar a temperatura e de iluminação no local de preparação

4. Use a prescrição médica durante a preparação

5. Realizar a preparação em uma superfície de trabalho seca

6. Desinfetar a superfície de trabalho com a solução estabelecida pela ComissãodeControledelnfecçãoHospitalar

7. Desinfetar a bandeja onde os medicamentos preparados serão colocados

8. Use equipamento de protecção pessoal de acordo com os protocolos institucionais

9. Realizar o procedimento com outro profissional de enfermagem, no caso de medicamentos potencialmente perigosos, para estabelecer duplo controlo

10. Desinfetar frascos ou ampolas e / ou ampolas

11. Lavar as mãos ou usar álcool gel

12. Medicamentos separados individualmente, verificando nome, dosagem e data de validade

13. Consulte o protocolo para a preparação estabelecida pela unidade, afixada em local visível

14. Use o material que é compatível com a solução (set e garrafa administração polifix IV)

15. Alterar agulha após a preparação do medicamento

16. Proteja o êmbolo da seringa com a sua embalagem após o preparo

17. Remover o ar a partir da seringa, após a preparação com a agulha com bainha

18. Não preparar os medicamentos para vários pacientes ao mesmo tempo

19. Prepare os medicamentos imediatamente antes da sua administração

20. Inspecione a medicação em relação à sua integridade física e química

22. Identificar a medicação correcta com o nome do paciente completo, número de cama, data, tempo e via de administração, e o nome do profissional que preparou 